\title{
Trypanosoma cruzi Mexican Strains Differentially Modulate Surface Markers and Cytokine Production in Bone Marrow- Derived Dendritic Cells from C57BL/6 and BALB/c Mice
}

\author{
Cecilia Gomes Barbosa, ${ }^{1}$ Tamires Marielem Carvalho Costa, \\ Chamberttan Souza Desidério, ${ }^{2}$ Paula Tatiana Mutão Ferreira, ${ }^{2}$ Mariana de Oliveira Silva, ${ }^{1}$ \\ César Gómez Hernández $\mathbb{D}^{2}{ }^{2}$ Malú Mateus Santos, ${ }^{2}$ Rafael Obata Trevisan, ${ }^{2}$ \\ Weslley Guimarães Bovi $\left(\mathbb{D},{ }^{2}\right.$ Virmondes Rodrigues $\left(\mathbb{D},{ }^{2}\right.$ Juliana Reis Machado $\left(\mathbb{D},{ }^{3}\right.$ \\ Luiz Eduardo Ramirez, ${ }^{1}$ Carlo José Freire de Oliveira $\mathbb{D D}^{2},{ }^{2}$ and Marcos Vinícius da Silva $\mathbb{D}^{1}$ \\ ${ }^{1}$ Laboratory of Parasitology, Department of Immunology, Microbiology and Parasitology, Federal University of Triângulo Mineiro, \\ Uberaba, MG, Brazil \\ ${ }^{2}$ Laboratory of Immunology, Department of Immunology, Microbiology and Parasitology, Federal University of Triângulo Mineiro, \\ Uberaba, MG, Brazil \\ ${ }^{3}$ Department of General Pathology, Federal University of Triângulo Mineiro, Uberaba, MG, Brazil
}

Correspondence should be addressed to Marcos Vinícius da Silva; marcosuftm@gmail.com

Received 16 March 2019; Revised 8 June 2019; Accepted 29 July 2019; Published 15 September 2019

Guest Editor: Luisa Cervantes-Barragan

Copyright (C) 2019 Cecilia Gomes Barbosa et al. This is an open access article distributed under the Creative Commons Attribution License, which permits unrestricted use, distribution, and reproduction in any medium, provided the original work is properly cited.

\begin{abstract}
Dendritic cells (DCs) are a type of antigen-presenting cells that play an important role in the immune response against Trypanosoma cruzi, the causative agent of Chagas disease. In vitro and in vivo studies have shown that the modulation of these cells by this parasite can directly affect the innate and acquired immune response of the host in order to facilitate its biological cycle and the spreading of the species. Many studies show the mechanisms by which T. cruzi modulates DCs, but the interaction of these cells with the Mexican strains of T. cruzi such as Ninoa and INC5 has not yet been properly investigated. Here, we evaluated whether Ninoa and INC5 strains evaded the immunity of their hosts by modulating the biology and function of murine DCs. The CL-Brener strain was used as the reference strain. Herein, it was demonstrated that Ninoa was more infective toward bone marrow-derived dendritic cells (BMDCs) than INC5 and CL-Brener strains in both BMDCs of BALB/c and C57BL/6 mice. Mexican strains of T. cruzi induced different cytokine patterns. In BMDCs obtained from BALB/c mice, Ninoa strain led to the reduction in IL-6 and increased IL-10 production, while in C57BL/6 mice Ninoa strain considerably increased the productions of TNF- $\alpha$ and IL-10. Also, Ninoa and INC5 differentially modulated BMDC expressions of MHC-II, TLR2, and TLR4 in both BALB/c and C57BL/6 mice compared to Brazilian strain CL-Brener. These results indicate that T. cruzi Mexican strains differentially infect and modulate MHC-II, toll-like receptors, and cytokine production in DCs obtained from C57BL/6 and $\mathrm{BALB} / \mathrm{c}$ mice, suggesting that these strains have developed particular modulatory strategies to disrupt DCs and, consequently, the host immune responses.
\end{abstract}

\section{Introduction}

Chagas disease, an illness identified 110 years ago by the physician and researcher Carlos Chagas, is a serious public health problem, affecting approximately 8 million people worldwide
$[1,2]$. Trypanosoma cruzi, the etiological agent of the disease, presents intraspecific genetic and phenotypic heterogeneity. Based on phylogenetic, molecular, biochemical, and biological markers, the causative agent of the disease is grouped into six discrete typing units (DTUs) ranging from TcI to TcVI 
[3-5]. The genetic diversity of T. cruzi exerts influence on the biological, clinical, immunological, and epidemiological variation of the disease, and it is also directly related to the establishment of the infection $[6,7]$. For example, the TcVI genotype is associated with human Chagas disease in countries of South America, especially north of the equator where several cases of human infection have been reported [4]. Specifically, the CL-Brener strain [8] belongs to the TcVI genotype, and the metacyclic forms derived from this strain are highly invasive in vitro and in vivo [9-11]. $\mathrm{TcI}$ is considered a homogeneous group but contains the largest distribution among the described genotypes and is subdivided into five subgroups (TcIa-TcIe) [5, 12-15]. TcI is the genotype that predominates in Mexico and is responsible for causing most of the clinical manifestations of Chagas disease. The Mexican strain of this genotype has different biological characteristics such as growth, metacyclogenesis, and infectivity in vitro and can cause patent and subpatent parasitemia. However, the strains belonging to the same TcI genotype differ in their ability to invade cells and cause infection [16-21]. Experimental studies have shown that although the Mexican strains belong to the same TcI genotype, they present differences in the induction of mortality (0-100\%), muscle cell tropism (mainly skeletal and cardiac), and in the inflammatory process generated by the infection. This indicates that biological behavior varies between these strains in the same DTU $[16,17,19,20]$. Thus, elucidating the underlying mechanisms that generate so many differences even in strains of the same genotype and in the same geographical region is essential for understanding the disease in Mexico.

The T. cruzi parasite presents three morphological forms in its biological cycle, and of these, metacyclic trypomastigotes are the infective forms eliminated by triatomines during blood feeding $[9,16,22-24]$. The surface molecules presented by the metacyclic trypomastigotes are fundamental for the interaction of the parasite with the host, and through these surface molecules, the protozoan can be recognized by host defense cells. In this context, dendritic cells (DCs) are one of the preferred targets of the infecting forms of T. cruzi [25]. Because of their efficient antigen presentation ability, DCs can detect pathogens and initiate an effective response through a cascade of triggered events that culminates in the presentation of antigen to lymphocytes and activation of a specific and protective immune response [26]. In this process, these cells are activated and direct the host immune response depending on the production of cytokines and the presence and intensity of surface markers that characterize their maturation [27-29]. During antigen presentation, these cells have high expression of molecular markers such as CD80, CD86, and MHC [27, 30-32]. Additionally, cellular migration markers such as CCR7, which are fundamental in the migration process of these cells to the presentation sites, are expressed [27, 33]. Furthermore, various proinflammatory cytokines such as IL-1 $\beta$, IL-12, IL-8, TNF- $\alpha$, and IL- 6 are synthesized and assist in the formation of a specific pattern of immune response [27, 34, 35].

The interaction with $T$. cruzi induces inhibition of the expression of important cell activation and cellular matura- tion markers such as CD80, CD86, MHC, and CD40 [36]. In addition, T. cruzi induces a DC death marker called PDL-1 that inhibits the production of proinflammatory cytokines such as IL-12, TNF- $\alpha$, and IL-6 and stimulates the synthesis of anti-inflammatory cytokines such as IL-10 and TGF- $\beta$ targeting a tolerogenic profile where there is less activation of $T$. cruzi-specific T lymphocytes $[6,25,28,37$, 38]. These immunomodulations by the parasite can vary depending on the T. cruzi strain and how they interact with these cells, highlighting the key role of DCs in the development of clinical forms of the disease [6, 7, 37, 39-43]. Although many studies have elucidated the mechanisms by which T. cruzi modulates DCs, the interaction of these cells with Mexican strains has not yet been properly investigated. The present study is aimed at investigating the infectivity, expressions of standard recognition receptors and costimulatory molecules, and the production of cytokines in DCs cultured with Mexican strains to understand how they evade the immune responses of their hosts by modulating these cells.

\section{Material and Methods}

2.1. Parasites. Two strains of T. cruzi from Mexico were used. They were maintained in the laboratory of parasitology of the Federal University of the Triângulo Mineiro, UFTM. Ninoa strain (MHOM/MX/1994) [44] was obtained from xenodiagnosis of a patient with acute Chagas disease, while the INC5 strain (MHOM/MX/1994) [45] was isolated from a patient with Chagasic cardiomyopathy $[19,20]$. CL-Brener is a Brazilian strain and was used as a reference for the study. This strain was isolated from a Triatoma infestans, belonging to the Department of Parasitology of the Federal University of São Paulo, UNIFESP, and was kindly provided by Dra. Nobuko Yoshida. The parasites were cultured at $28^{\circ} \mathrm{C}$ in liver infusion tryptose (LIT) medium supplemented with $10 \%$ fetal bovine serum. Metacyclic forms of cultures in stationary growth phase were purified by column passing DEAEcellulose as previously described [46].

\subsection{Animals and Differentiation of Bone Marrow-Derived} DCs (BMDCs). Male BALB/c and C57BL/6 (6-8 weeks old) wild-type mice were bred and maintained in experimental animal facilities of the Federal University of Triângulo Mineiro, UFTM, Uberaba, MG, Brazil, according to the guidelines of the Ethics Committee on Animal Use (CEUA). All the experiments were conducted according to the Ethics Committee on the Use of Animals of the Federal University of Triângulo Mineiro. Bone marrow cells from the femurs and tibiae removed from mice were centrifuged at $400 \times g$ for $10 \mathrm{~min}$ at $8^{\circ} \mathrm{C}$ in RPMI 1640 medium (GE Healthcare, Uppsala, Sweden). Subsequently, $2 \mathrm{~mL}$ of lysis buffer was added for lysing the red cells, and the cells were washed thrice for counting. Cells were counted in a Neubauer chamber and resuspended to $5 \times 10^{6}$ cells/mL in RPMI 1640 medium with the addition of $50 \mathrm{mM}$ Hepes (Gibco, Grand Island, NY, USA), $10 \%$ of inactivated fetal bovine serum (Gibco, USA), 2 mM L-glutamine (Gibco, USA), $40 \mathrm{mg} / \mathrm{mL}$ gentamicin, and $12.5 \mathrm{ng} / \mathrm{mL}$ murine GM-CSF (BD) at $37^{\circ} \mathrm{C}$ 
in a humidified atmosphere with $5 \% \mathrm{CO}_{2}$. On day $3,10 \mathrm{~mL}$ of RPMI medium containing $12.5 \mathrm{ng} / \mathrm{mL}$ GM-CSF was added. Cells were further differentiated for additional 4 days with GM-CSF containing complete medium. After 7 days of culture, the cells were collected and analyzed by flow cytometry to determine the percentage of CD11b and CD11c, and further experiments were performed only after evaluating this percentage. All cultures presented at least $80 \%$ of $\mathrm{CD}_{11} \mathrm{~b}^{+} \mathrm{CD} 11 \mathrm{c}^{+} \mathrm{DCs}$, and bone marrow-derived dendritic cells (BMDCs) were harvested and cultured in 96-well plates.

2.3. In Vitro Infection of DCs. After 7 days of differentiation, BMDCs at a concentration of $1.5 \times 10^{5}$ cells per well (96well plate) in $250 \mu \mathrm{L}$ of $10 \%$ RPMI 1640 medium were incubated for $18 \mathrm{~h}$ with the three different T. cruzi strains (CL-Brener, Ninoa, or INC5 at MOI 2:1) with or without LPS $(5 \mu \mathrm{g} / \mathrm{mL})$. Cells were then evaluated for parasite infection using $4^{\prime}$,6-diamidino-2-phenylindole dihydrochloride (DAPI; Molecular Probes, Eugene, Oregon, US). Expression of MHC-II, CD80, CD86, TLR2, and TLR4 surface markers was evaluated by flow cytometry, and the production of TNF- $\alpha$, IL-10, IL-12p70, CCL-2, and IL- 6 was measured by cytometric bead array. A MOI of $2: 1$ was chosen because this ratio of cell and parasite was sufficient to affect the different steps of the DC biology or to assess parasitic infectivity.

2.4. Determination of T. cruzi-Infected DCs. BMDCs in 96well plates were analyzed by fluorescence microscopy using DAPI. BMDCs differentiated from both the mouse strains were cultured with different strains of T. cruzi, harvested, and incubated at $4^{\circ} \mathrm{C}$ for $30 \mathrm{~min}$ with $10 \mu \mathrm{M}$ DAPI. Cells were washed twice with $\mathrm{PBS}$ and centrifuged at $400 \times \mathrm{g}$ at $4^{\circ} \mathrm{C}$ for $10 \mathrm{~min}$ and immediately analyzed by EVOS Cell Imaging System (Thermo Scientific, USA) at $\times 400$ magnification. The number of intracellular parasites was counted in a total of 100 cells, and percentage of infected cells, parasites per 100 cells, and mean parasite load in infected cells were determined.

2.5. Flow Cytometry Analysis. BMDCs differentiated from both mouse strains were analyzed by flow cytometry using the following monoclonal antibodies: anti-CD11c, antiMHC-II, anti-CD80, anti-CD86, anti-TLR2, or anti-TLR4, labeled with APC, FITC, PE, or PECy7 according to the intended purpose. The acquisition was obtained using an Accuri flow cytometer (BD Immunocytometry Systems), and the analyses were performed using the FlowJo software.

2.6. Cytometric Bead Array. Cytometric bead array was performed using the CBA Mouse Inflammation Kit (BD Biosciences) according to the manufacturer's instructions, and the following cytokines were measured: TNF- $\alpha$, IL-10, IL-12p70, IL-6, and chemokine CCL-2. Briefly, supernatants of BMDCs from C57BL/6 and BALB/c mice cultured with T. cruzi, with or without LPS, were incubated with beads coupled with specific monoclonal antibodies and PEconjugated secondary antibodies for $4 \mathrm{~h}$ at room temperature. Beads were washed and the acquisition was performed using the Accuri flow cytometer (BD Immunocytometry Systems). The concentration of the samples was estimated by comparing the PE fluorescence obtained from the standard curve obtained by serial dilution of recombinant murine cytokines. Results were analyzed by 5-parameter logistic regression with FCAP array software and expressed in $\mathrm{pg} / \mathrm{mL}$.

2.7. Statistical Analysis. The results were analyzed by GraphPad Prism 7.0 (GraphPad Software, San Diego, CA, USA). The Kruskal-Wallis test with a Dunn's post hoc test was performed for data with a non-Gaussian distribution. Bar graphs show mean and standard error of the mean. The results were considered significant when the $p$ value was lower than $0.05(5 \%)$.

\section{Results}

3.1. Evaluation of the Infectivity of Different Strains of T. cruzi in DCs Derived from BALB/c and C57BL/6 Mice. In general, the different $T$. cruzi strains were able to infect DCs. However, the percentage of infection varied depending on the strain and the origin of DCs $[6,7,37,47]$. In BMDCs derived from BALB/c mice, the percentage of cells infected with the different strains did not show a significant difference despite experiencing a slight increase when these cells were cultured with LPS (Figure 1(a)). Contrastingly, when evaluating the amount of parasites present in a total of 100 BMDCs, we found that cells cultured with T. cruzi from the Ninoa strain previously stimulated with LPS exhibited a significant increase in the number of parasites compared to the INC5 strain plus LPS $(p=0.0427)$ (Figure $1(b))$. The same profile in relation to the Ninoa and INC5 strains plus LPS was found by analyzing the amount of parasites present in each infected cell $(p=0.0219)$ (Figure $1(c))$. In the CL-Brener strain without LPS treatment, the number of parasites per infected cell was significantly higher compared to the INC5 strain also without LPS treatment $(p=0.0427)$ (Figure $1(c))$. When the BMDCs derived from $\mathrm{C} 57 \mathrm{BL} / 6$ mice were evaluated, the percentage of infected cells after culture with CL-Brener strain plus LPS presented a significant variation $(p=0.0234$ ) compared to BMDCs cultured with INC5 strain plus LPS (Figure 1(d)). The amount of parasites per 100 cells showed a significant increase when the CL-Brener strain was compared to the INC5 strain $(p=0.0422)$. The same difference in infectivity was found when the number of parasites in BMDCs cultured with the CL-Brener strain plus LPS was compared to BMDCs cultured with the Ninoa strain plus LPS $(p=0.0230)$ (Figure $1(\mathrm{e}))$. The same pattern was observed in the amount of parasites per infected cell comparing the effects of the Ninoa and CL-Brener strains $(p=0.0181$ ) (Figure 1(f)). In Figure 1(g), we show a representative picture of DAPI labeling evidencing the infectivity of different strains of T. cruzi in BMDCs in vitro.

3.2. Production of Cytokines and Chemokine CCL-2 in DCs Cultured with Different Strains of T. cruzi. The concentration of cytokines in BMDCs from BALB/c mice did not show statistically significant differences in any of the analyses, regardless of whether they were infected only with the strains or in the presence of LPS. The following cytokine levels were 


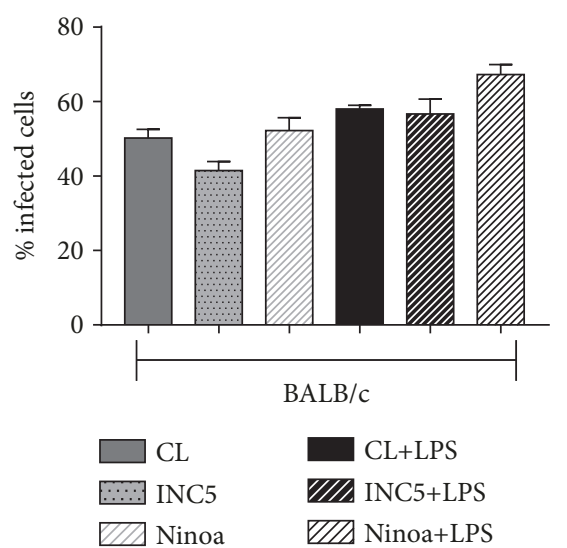

(a)
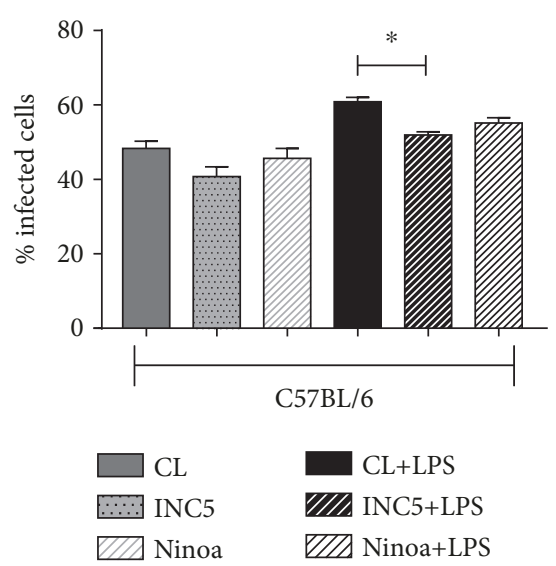

(d)

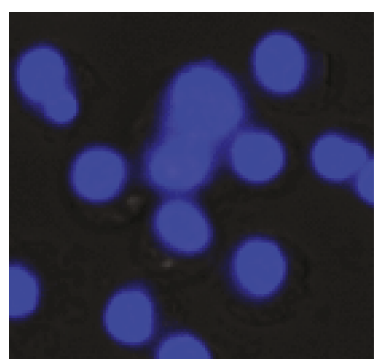

BMDC

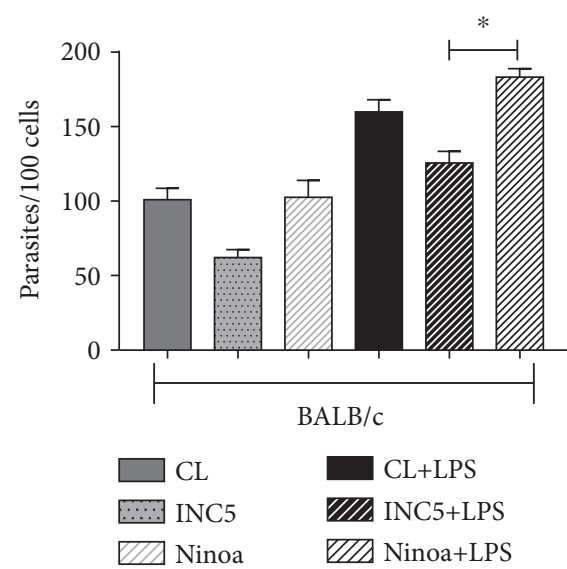

(b)

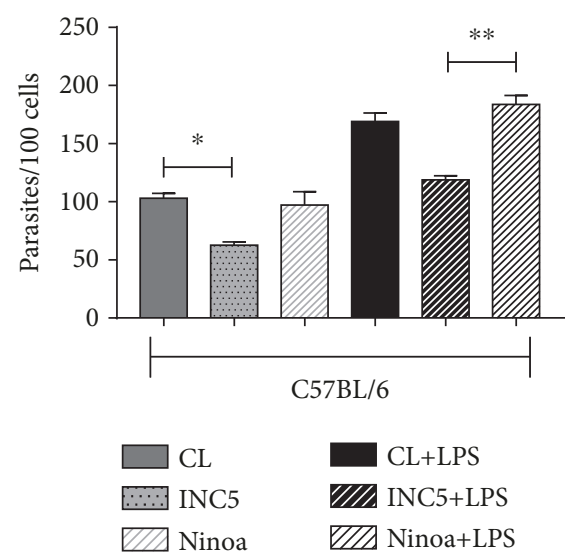

(e)

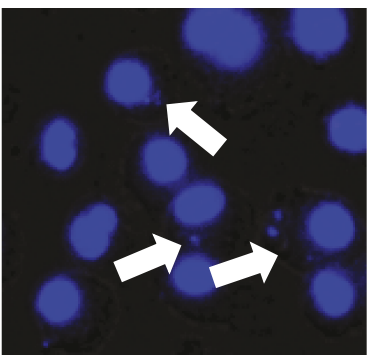

BMDC+LPS+CL Brener

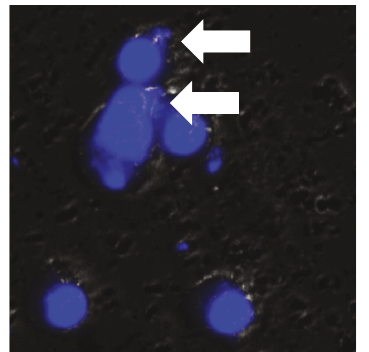

BMDC+LPS+INC5

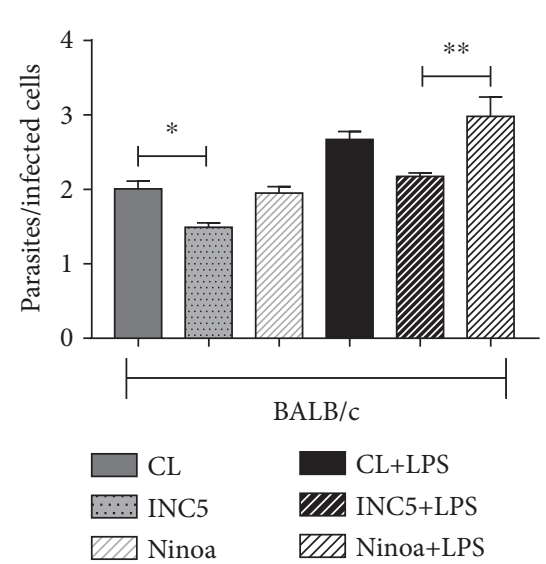

(c)
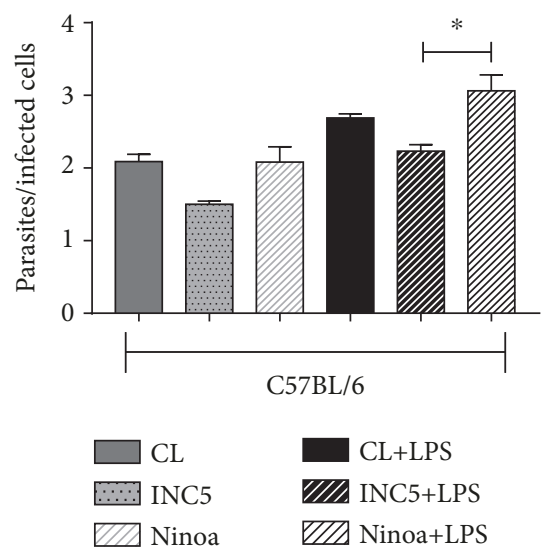

(f)

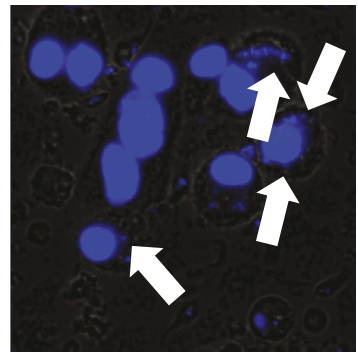

BMDC+LPS+Ninoa

(g)

FIgURE 1: Trypanosoma cruzi infectivity in murine BMDCs from BALB/c and C57BL/6 mice. BMDCs with or without LPS stimulation $(5 \mu \mathrm{g} / \mathrm{mL}$ ) were incubated for $18 \mathrm{~h}$ with different T. cruzi strains (MOI 2:1) and stained with DAPI. (a) and (d) show the percentage of infected cells; in (b) and (e) the number of parasites per 100 cells and in (c) and (f) the ratio of parasites per infected cell are shown. (g) Representative images of BMDCs infected with T. cruzi after stimulation with LPS $(5 \mu \mathrm{g} / \mathrm{mL})$ and stained with DAPI. Statistical analysis, when applicable, was performed with the Kruskal-Wallis test with Dunn's posttest, where ${ }^{* * * *} p<0.05$.

measured: TNF- $\alpha$, IL-6, IL-12p70, CCL-2, and IL-10 (Figures 2(a)-2(e)). When we analyzed the cytokines present in the culture supernatant of BMDCs derived from C57BL/6 mice, a significant increase was observed in the production of TNF- $\alpha$ in BMDCs cultured with the Ninoa strain without or plus LPS compared to cultures without T. cruzi infection $(p=0.0094)$ (Figure 3(a)). Also, the Ninoa strain induced a significant increment of IL-10 production in LPS-treated cultures compared to LPS alone and all other strains plus LPS ( $p=0.00001$ for LPS, $p=0.023$ for CL-Brener, and $p=0.0331$ for INC5) (Figure 3(e)). We did not find significant differences in the production of IL-6 (Figure 3(b)), IL-12p70 (Figure 3(c)), and CCL-2 (Figure 3(d)).

3.3. Percentage of Cytokine and Chemokine CCL-2 Variation in DCs Cultured with Different Strains of T. cruzi. In order to demonstrate how the different $T$. cruzi strains could modulate the ability of dendritic cells to produce cytokines, we calculated the variation of cytokines by determination of percentage of increment or reduction in cytokines produced 
by T. cruzi-infected BMDCs previously treated with LPS and those maintained only with LPS $(5 \mu \mathrm{g} / \mathrm{mL})$. Globally, the interaction of BMDCs with T. cruzi induced TNF- $\alpha$, IL-6, CCL-2, and IL-10 increment and IL-12p70 reduction. The cytokine IL-6 (Figure 4(b)) showed significant alteration only in the BMDCs derived from $\mathrm{BALB} / \mathrm{c}$ mice, showing a reduction in cells stimulated with the Ninoa strain compared to cells stimulated with the CL-Brener strain $(p=0.0387)$. On the other hand, the Ninoa strain induced significant increment in CCL-2 compared to CL-Brener in BALB/C mice $(p=0.01)$. IL-10 also showed significant variation for both mice strains, as BMDCs cultured with the Ninoa strain had an increase in IL-10 compared to BMDCs cultured with the INC5 strain in BALB/c mice $(p=0.0219)$ and compared to INC5 and CL-Brener in C57BL/6 mice $(p<0.0001)$. We did not observe any significant changes in the variation of TNF- $\alpha$ (Figure 4(a)) and IL-12p70 among strains (Figure 4(c)). In Figures 4(f) $-4(\mathrm{i})$, it is possible to observe the variation in the production of the cytokines according to the different strains with or without the addition of LPS.

3.4. Evaluation of MHC-II, CD80, CD86, TLR2, and TLR4 Expression in DCs Cultured with Different Strains of $T$. cruzi. Data related to flow cytometry were analyzed by ascertaining the variation of the percentage of positive cells and the variation of the expression per cell (mean fluorescence intensity). Our results point that T. cruzi Mexican strains significantly reduce cells expressing MHC-II, TLR2, and TLR4 compared to the Brazilian strain CL-Brener. Specifically, the interaction with INC-5 and Ninoa led to a lower MHC$\mathrm{II}^{+}$BMDC in both BALB/c $(p=0.0011)$ and $\mathrm{C} 57 \mathrm{BL} / 6$ $(p=0.0004)$ mice (Figure 5(a)). The costimulatory molecules CD80 and CD86 presented a negative variation in BMDCs cultured with all evaluated (Figures 5(b) and 5(c)). For both $\mathrm{BALB} / \mathrm{C}$ and $\mathrm{C} 57 \mathrm{BL} / 6$, the CL-Brener strain induced higher percentage of TLR2 ${ }^{+}$BMDCs compared to the INC5 strain $(p=0.0051$ and $p=0.0001$, respectively) and to Ninoa $(p=0.0051$ and $p=0.0001$, respectively, Figure 5(d)). In a similar way, the CL-Brener strain interaction induced a higher percentage of TLR4 ${ }^{+}$BMDCs in both mice strains compared to the INC5 $(p=0.0001$ for $\mathrm{BALB} / \mathrm{c}$ and C57BL/6) and Ninoa strains $(p=0.0001$ for $\mathrm{BALB} / \mathrm{c}$ and C57BL/6) (Figure 5(e)).

In both mouse strains, the INC5 strain led to a significant increment in BMDC MHC-II expression compared to the CL-Brener strain $(p=0.0319)$ and Ninoa $(p=0.0004)$ in $\mathrm{BALB} / \mathrm{c}$. Interestingly, while in BALB/c mice the CL-Brener strain induced a slight increment in MHC-II, in C57BL/6 mice, this expression was inhibited and significantly different from INC5 and Ninoa $(p=0.0427$, Figure 6(a)). All $T$. cruzi strains induced an inhibition in CD80 expression for both mouse strains (Figure 6(b)). For CD86, only Ninoa induced a slight increment in expression but without statistical significance (Figure 6(c)). The CL-Brener strain also induced a considerable increment in TLR2 expression in both mouse strains compared to the INC5 and Ninoa strains $(p=0.0002$ for $\mathrm{BALB} / \mathrm{c}$ and $p=0.0001$ for C57BL/6, Figure 6(d)). On the other hand, the CL-Brener strain infec- tion induced a lower expression of TLR4 $4^{+}$BMDCs compared to the INC5 and Ninoa strains $(p=0.0004$ for BALB/c and $p=0.01$ for C57BL/6, Figure 6(d)). Radar plots present a summary of the impact of the interaction with different $T$. cruzi strains in LPS-stimulated BMDCs from BALB/c (Figure 6(f)) and C57BL/6 (Figure 6(g)) mice.

\section{Discussion}

Our study is aimed at evaluating the infectivity and immunomodulatory capacity of different Mexican strains of T. cruzi on DCs derived from BALB/c and C57BL/6 mice. Our results point to the fundamental role of these strains in the interaction with these DCs during the in vitro coculture, depending on the mouse lineage.

The biological behavior, anatomical route of invasion, inoculum, surface molecules expressed in the metacyclic forms of T. cruzi, and host immune response are factors that are closely related to the establishment of the infection $[6,9$, 48-50]. Our data showed that all three strains infected DCs efficiently. However, strains belonging to the TcI genotype (AQ1.7, Mutum, and G) presented a profile with low infectivity in BMDCs $[6,51]$. This can be explained by the fact that $T$. cruzi presents a high intraspecific genetic and phenotypic diversity, especially for the TcI genotype. Thus, different genetic markers indicate that there is an intra-DTU genetic variation, and the $\mathrm{TcI}$ is the genotype that presents high genetic heterogeneity. This may be related to the different epidemiological characteristics and generate controversies regarding the infectivity and pathogenicity of the strains belonging to this genetic group $[4,13,19,52]$.

When inoculated in Swiss mice, the parasites of the INC5 and Ninoa strains presented patent parasitemia, high infectivity, and mortality. Intense tissue parasitism was observed in several organs of the experimental animals, increasing the virulence of these strains [20, 52]. However, trypomastigote forms were used in these other studies [17, 20, 21]. Notably, the different evolutionary forms of $T$. cruzi use different strategies in adhesion or invasion of cells because of the specific stage molecules, which affect the infectivity and pathogenicity, even in the same strain $[53,54]$. The parasite inoculation pathway can also influence the biological behavior of the strain because of the different biological barriers that the parasite needs to overcome to establish the infection, directly impacting the immune response and host resistance [50]. One factor that needs to be considered is the type of cell or tissue that the metacyclic form infects. The Ninoa strain showed low parasitemia and mortality and a mild inflammatory process when inoculated orally in mice of this same strain. In oral infection, the parasites enter into the epithelial cells of the stomach and can undergo pepsin action, and the metacyclic trypomastigote forms express on their surface molecules that can facilitate or inhibit this invasion process. Recent studies have shown that the TcI genotype has a poor infective oral profile, as they express gp90 on their surface, and this molecule negatively regulates invasion to the target cell $[16,55]$. 

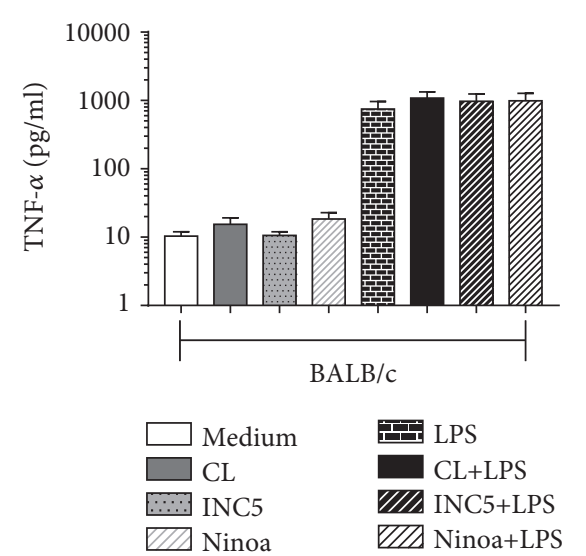

(a)

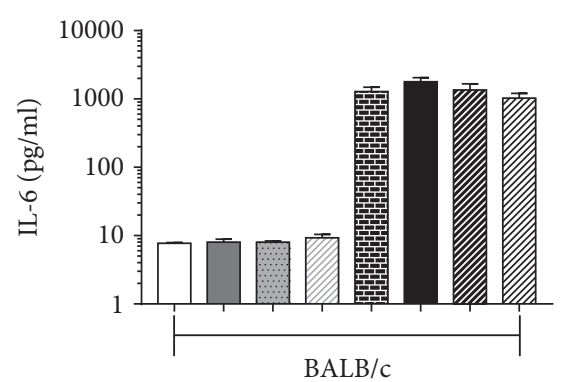

LPS

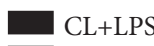

VIII INC5+LPS

VIIA Ninoa+LPS

(b)

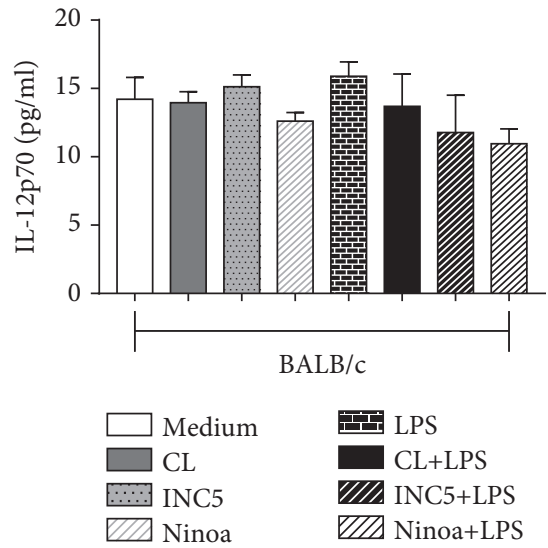

(c)

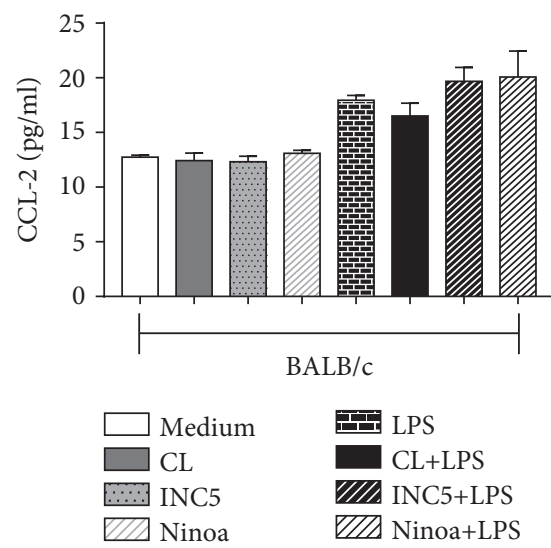

(d)

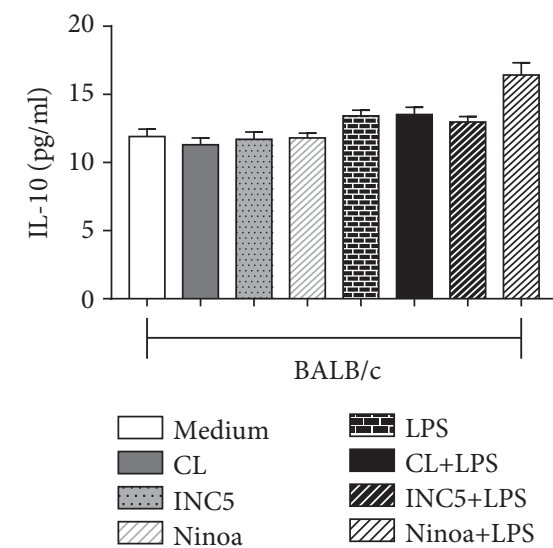

(e)

FIGURE 2: Cytokine and chemokine production by BALB/c-derived BMDCs infected with CL-Brener, Ninoa, and INC5 strains of T cruzi. BMDCs with or without LPS stimulation $(5 \mu \mathrm{g} / \mathrm{mL})$ were incubated for $18 \mathrm{~h}$ with different $T$. cruzi strains (MOI $2: 1)$, and the production of cytokines and chemokine was evaluated by CBA: (a) TNF- $\alpha$, (b) IL-6, (c) IL-12p70, (d) CCL-2, and (e) IL-10. Statistical analysis, when applicable, was performed with the Kruskal-Wallis test with Dunn's posttest, where ${ }^{*, * *} p<0.05$.

On the other hand and consistent with our present study, the CL-Brener strain presents a highly infectious profile in in vitro studies, and its profile is well studied in oral infection. An explanation for the increased infectivity may be the glycoprotein gp82 expressed on its surface that facilitates the entry of the parasite into the cell through the mobilization of intracellular calcium $[9,11]$. These different results using the same strains show that in addition to the parasite genetics, the infected cell line and the immune response caused by the contact of the parasite with the cell are associated with the infection. Additionally, the cells derived from the C57B/6 and $\mathrm{BALB} / \mathrm{c}$ mice present different degrees of susceptibility and/or resistance [51]. Our results show that the Ninoa strain has a higher infectivity potential when the BMDCs are activated with LPS, while the CL-Brener strain is more infective in BMDCs not yet activated. Different strains have shown distinct infectivity potentials [6], and this can be related to the ability of these cells to recognize the molecules presented on the surface of the parasite [56]. In addition, in BALB/c mice, we observed a similar cell interaction for all strains, while in C57BL/6 the CL-Brener strain had a better perfor- mance. This suggests that a strain can have different behaviors depending on the mice used, as shown in experiments with Y strain [57].

Once the ability of infection in cells derived from the two strains was confirmed, we analyzed the ability of these strains to modulate surface markers and stimulate the production of cytokines. We observed that both strains of T. cruzi and lineage of mice presented different patterns for the parameters evaluated. In BMDC from BALB/c mice, the Ninoa strain lead to a reduction in IL- 6 and an increase in IL-10 production, while in C57BL/6 mice the cytokine production was associated with a huge increment in TNF- $\alpha$ and IL-10. Cytokines are one of the most relevant factors in determining the course of infection $[17,51,58]$. During the process of infection by T. cruzi, a modulating response associated with susceptibility to the disease can be triggered by the parasite. For instance, in macrophages infected with T. cruzi, the activation of TLR is weak, leading to a decrease in the production of proinflammatory cytokines such as IL-12 [59]. In addition, the production of IL-10 and TGF- $\beta$ is stimulated in infected macrophages, and this causes a favorable response to the 

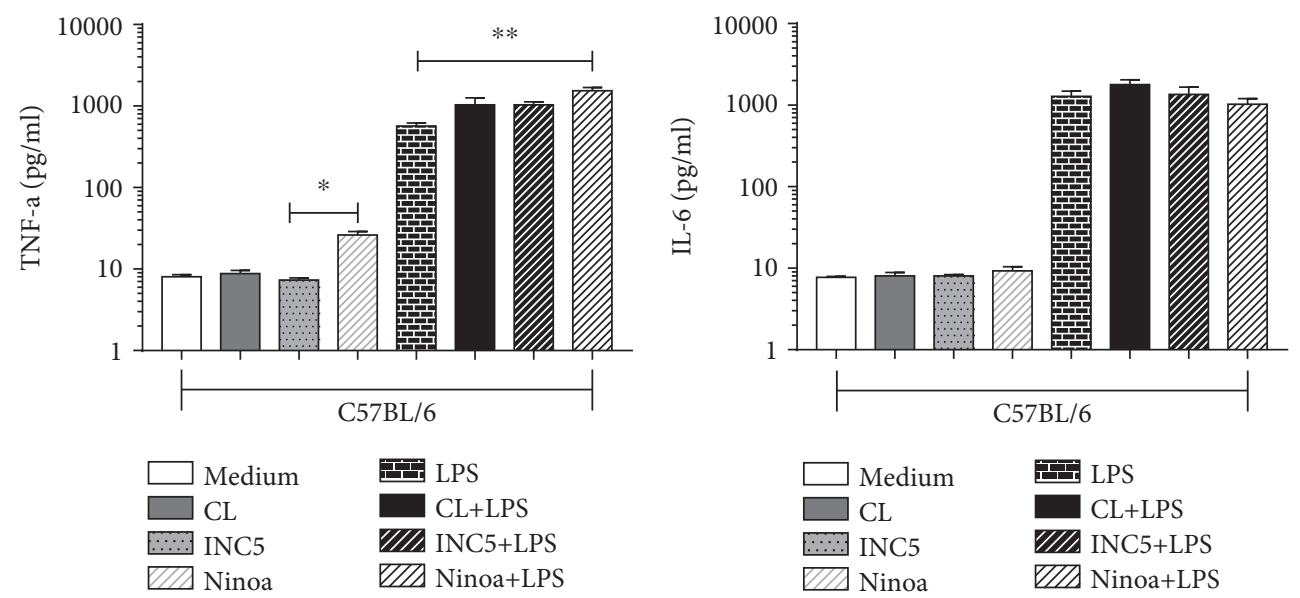

(a)

(b)
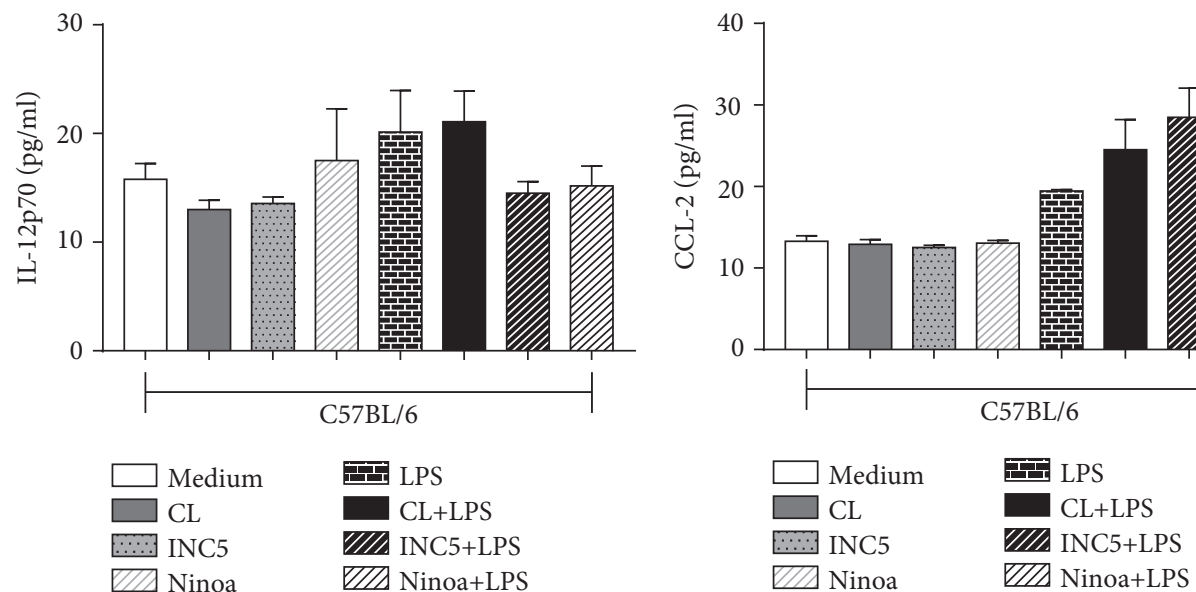

(d)

(c)

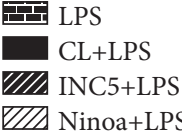

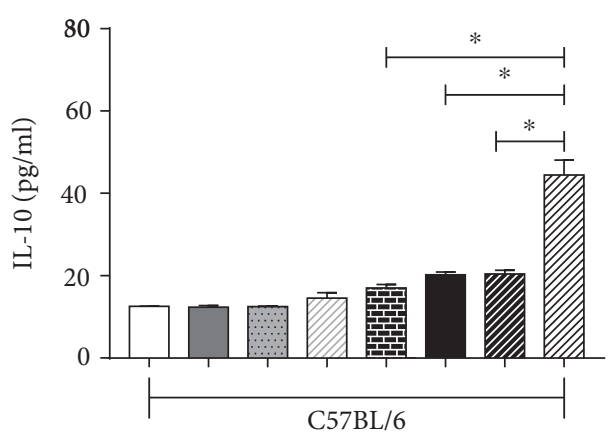

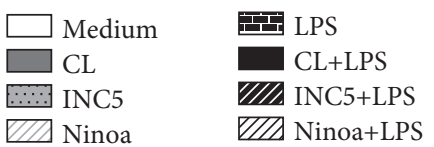

(e)

FIgure 3: Cytokine and chemokine production by C57BL/6-derived BMDC infected with CL-Brener, Ninoa, and INC5 strains of T cruzi. BMDCs with or without LPS stimulation $(5 \mu \mathrm{g} / \mathrm{mL}$ ) were incubated for $18 \mathrm{~h}$ with different $T$. cruzi strains (MOI $2: 1$ ), and the production of cytokines and chemokine was evaluated by CBA: (a) TNF- $\alpha$, (b) IL-6, (c) IL-12p70, (d) CCL-2, and (e) IL-10. Statistical analysis, when applicable, was performed with the Kruskal-Wallis test with Dunn's posttest, where ${ }^{* * *} p<0.05$. 


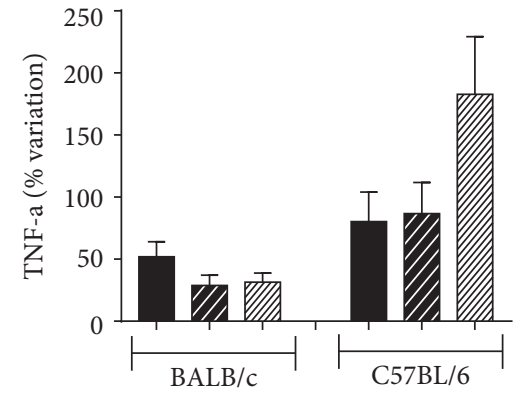

$\square \mathrm{CL}$

ZZ INC5

VIIA Ninoa

(a)

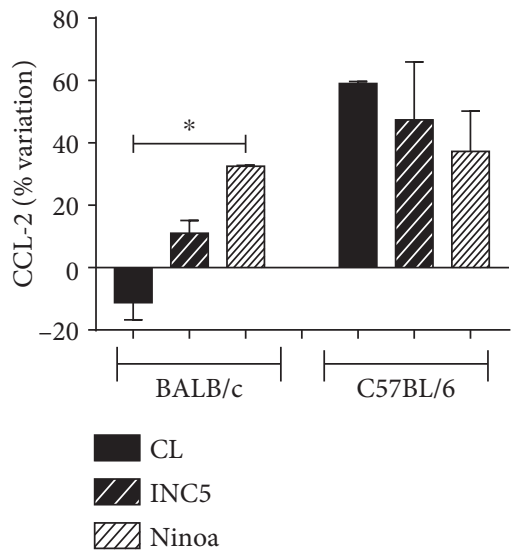

(d)

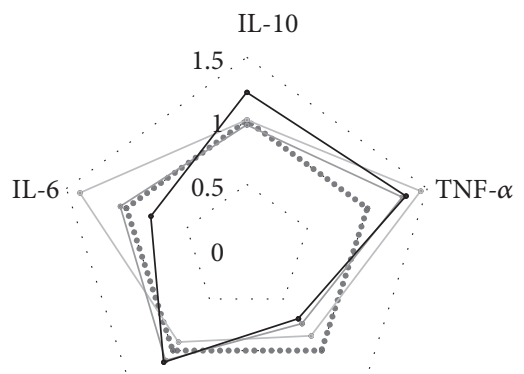

CCL-2

IL-12p70

$\mathrm{BALB} / \mathrm{c}$

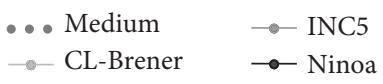

(g)

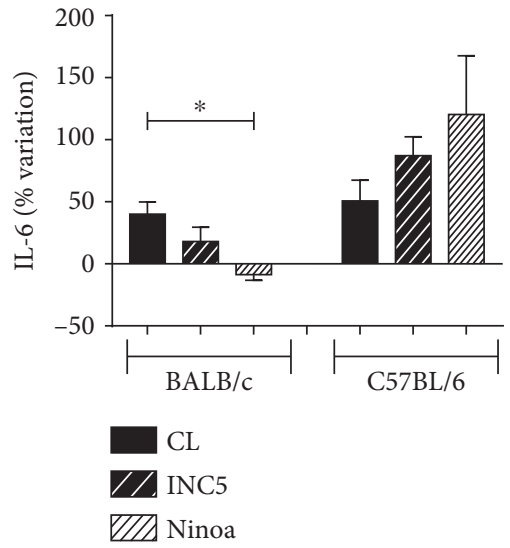

(b)

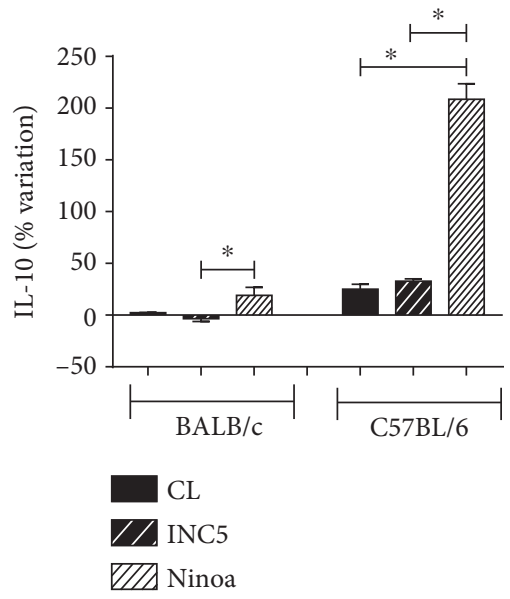

(e)
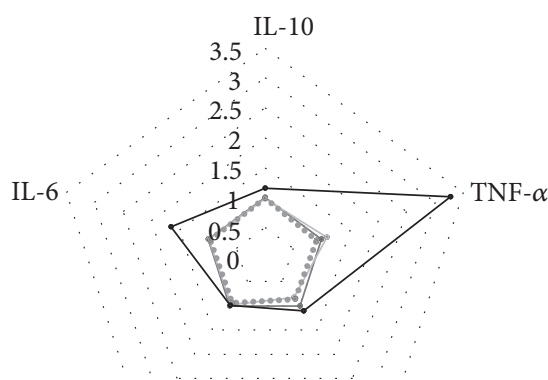

CCL-2

IL-12p70

C57BL/6

$\cdots$ Medium $\rightarrow$ INC5

(h)

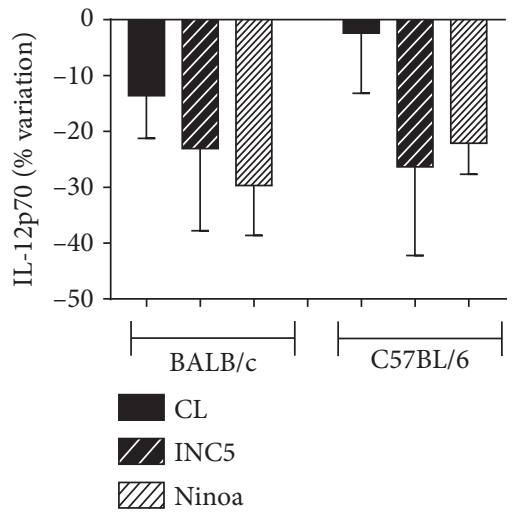

(c)

IL-10

2

1.5

IL-6

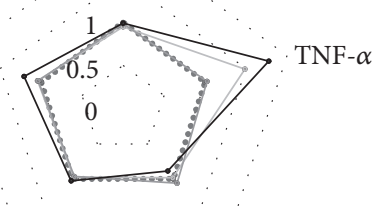

CCL-2

IL-12p70

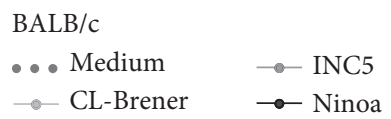

(f)

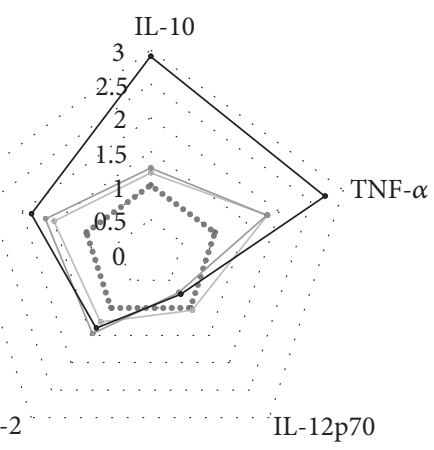

C57BL/6

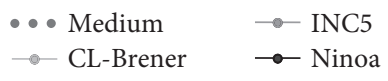

(i)

FIgURE 4: Percentage of variation in the levels of pro-and anti-inflammatory cytokines and CCL-2 chemokine in the supernatant of BMDCs of $\mathrm{BALB} / \mathrm{c}$ and $\mathrm{C} 57 \mathrm{BL} / 6$ mice. The variation of cytokines was calculated by determination of percentage of increment or reduction in cytokines produced by T. cruzi-infected BMDCs previously stimulated with LPS and those maintained only with LPS (5 $\mu \mathrm{g} / \mathrm{mL})$. (f, h) Representation of the radar graph of the cytokine pattern in the dendritic cell culture supernatant. The lines highlight the change in cytokine levels in dendritic cells infected with different strains (MOI 2:1) in relation to the medium only with dendritic cells. (g, i) The pattern of cytokines in the dendritic cell culture supernatant previously stimulated with LPS. Data were obtained by calculating the ratio between T. cruzi-infected cells and their respective control. Statistical analysis, when applicable, was performed with the Kruskal-Wallis test with Dunn's posttest, where ${ }^{*, * *} p<0.05$. 

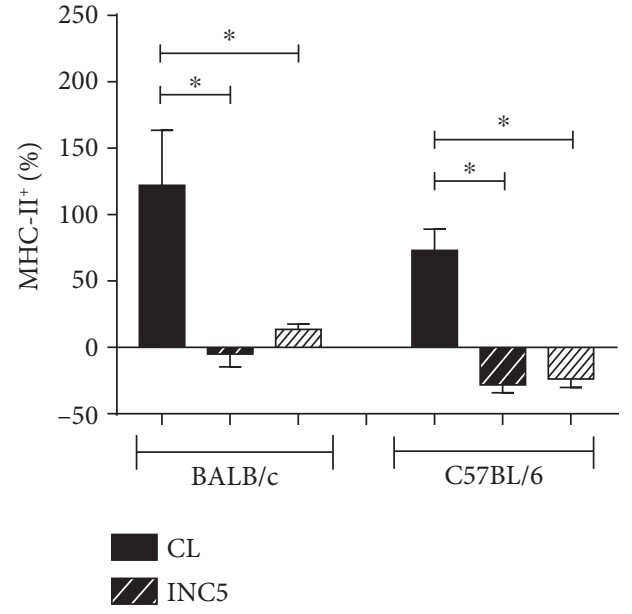

ZII Ninoa

(a)

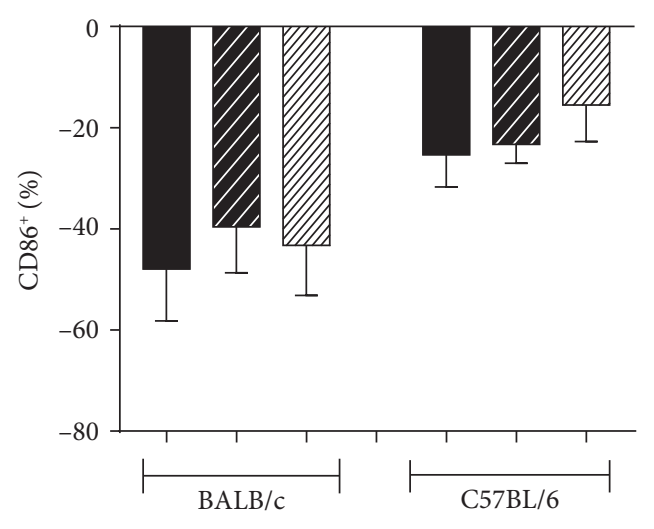

CL

ZIINC5

VIIA Ninoa

(c)

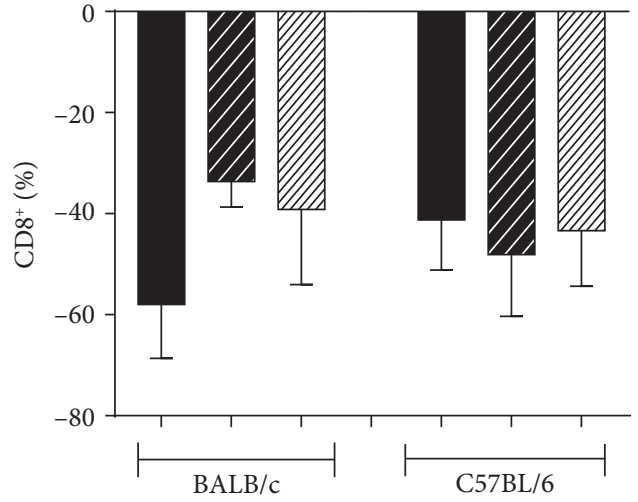

$\mathrm{CL}$

$7 /$ INC5

WII Ninoa

(b)

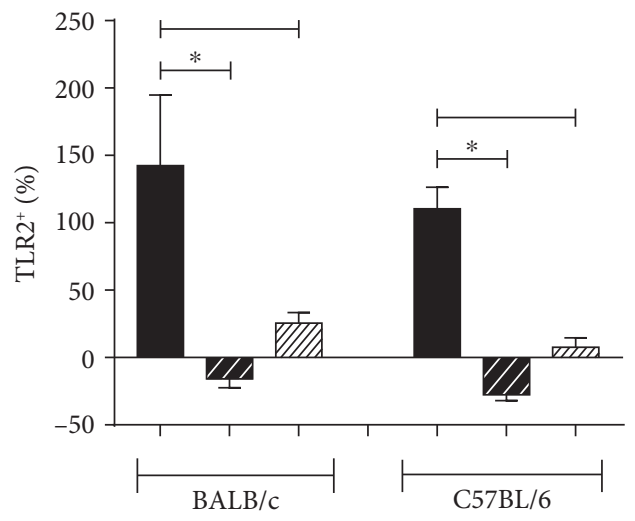

CL

ZZINC5

ZII Ninoa

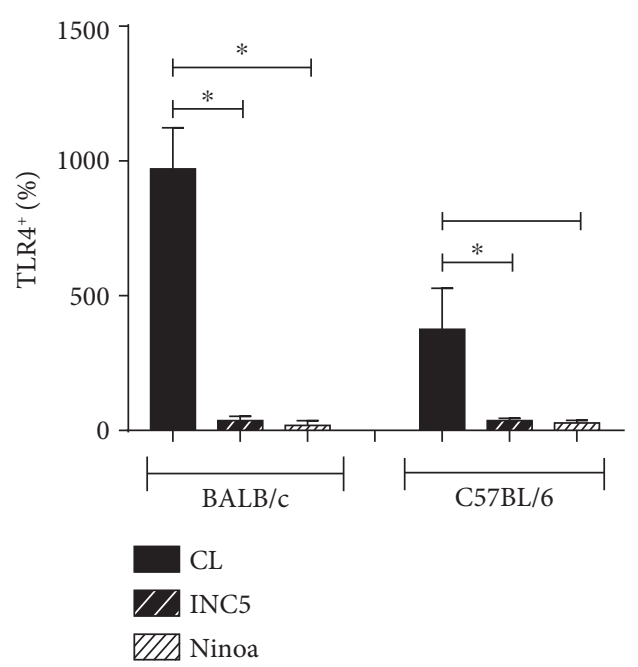

(e)

Figure 5: Percentage of BMDCs expressing MHC-II, costimulatory molecules, and toll-like receptors after in vitro T. cruzi infection. The expression of molecules was evaluated by flow cytometry and represented as a variation of the percentage of BMDCs expressing (a) MHCII, (b) CD80, (c) CD86, (d) TLR2, and (e) TLR4. Statistical analysis, when applicable, was performed with the Kruskal-Wallis test with Dunn's posttest, where ${ }^{*} p<0.05$. 


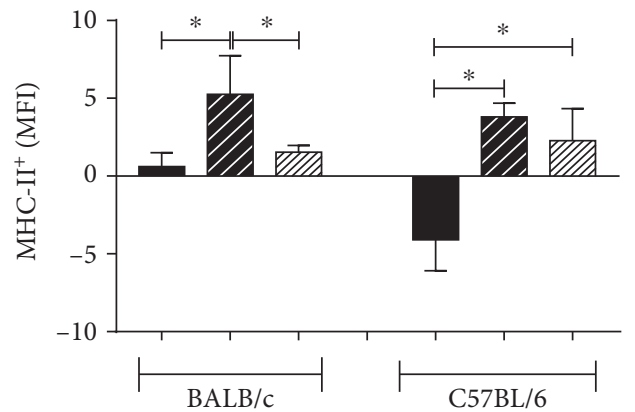

CL

$\square 】$ INC5

ZZI Ninoa

(a)

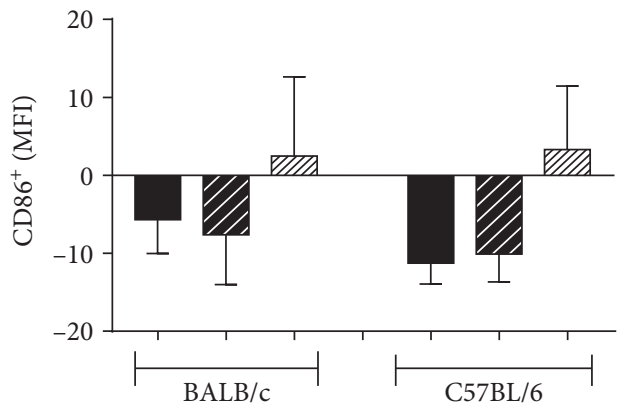

CL

$\measuredangle 】$ INC5

ZII Ninoa

(c)

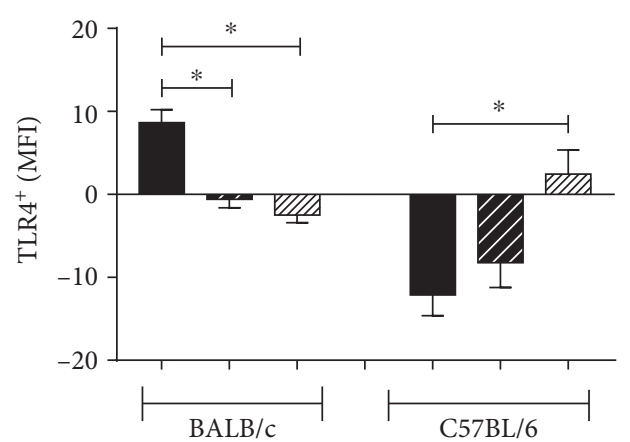

CL

$\measuredangle /$ INC5

VIIA Ninoa

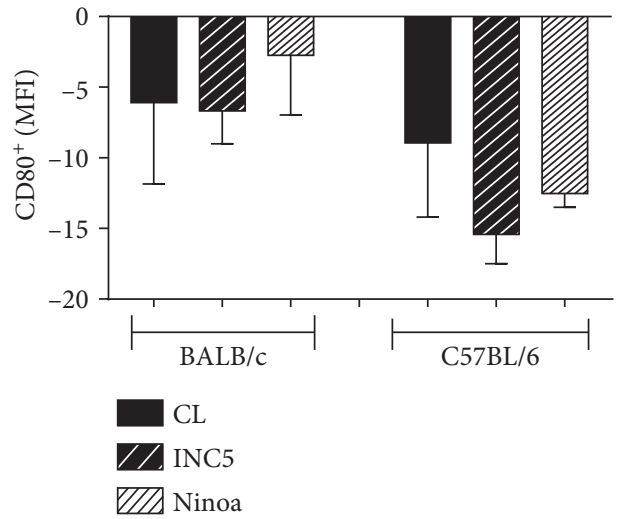

(b)

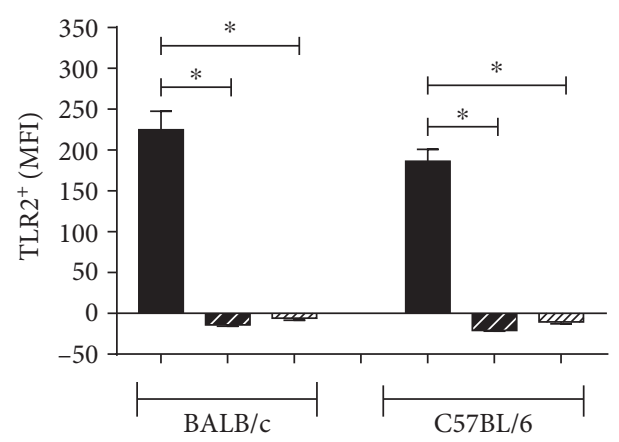

CL

$\angle \triangle$ INC5

ZII Ninoa

(d)

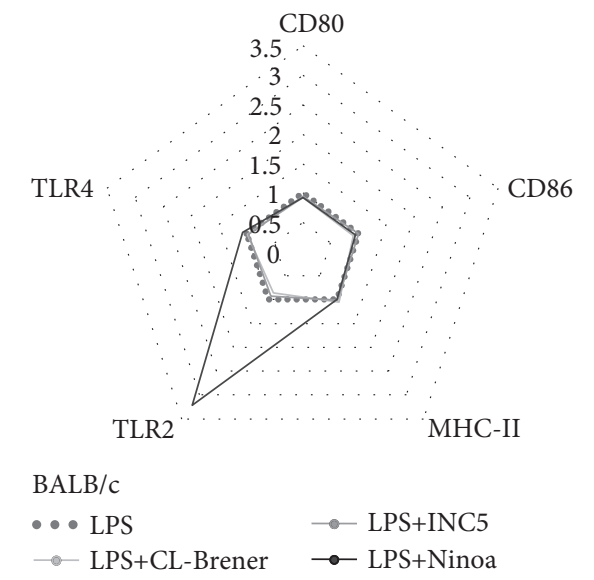

(e)

(f)

Figure 6: Continued. 


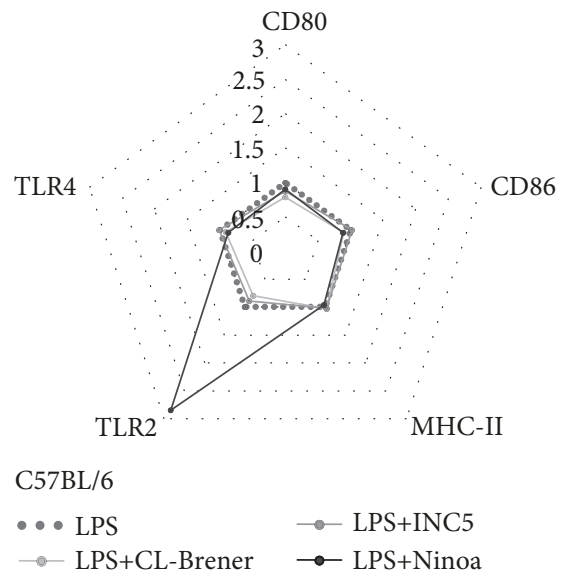

(g)

FIgURE 6: Expression of MHC-II, costimulatory molecules, and toll-like receptors in BMDCs after in vitro T. cruzi infection. The expression of molecules was evaluated by flow cytometry and represented as a variation of the mean intensity of fluorescence (a) MHC-II, (b) CD80, (c) CD86, (d) TLR2, and (e) TLR4. ( $\mathrm{f}, \mathrm{g})$ Representation of the cytokine secretion pattern in BMDC culture supernatant. The lines highlight the change in cytokine levels in LPS-stimulated BMDCs and infected with different strains of T. cruzi (MOI 2:1) in relation to uninfected LPS-stimulated BMDCs. Statistical analysis, when applicable, was performed with the Kruskal-Wallis test with Dunn's posttest, where ${ }^{*} p<0.05$.

parasite to be triggered $[37,60,61]$. Similar results were found in our study, where the Ninoa strain presented more modulatory behaviors compared to the other strains under similar conditions, especially when the cells were already activated. According to Gil-Jaramillo et al. [7], more virulent strains can manipulate DCs to produce more tolerogenic cytokines.

Ferreira et al. [51] described increased levels of IL-10 in $\mathrm{C} 57 \mathrm{~B} 1 / 6$ and $\mathrm{BALB} / \mathrm{C}$ mice in the acute phase of the disease. In addition, BALB/c mice had elevated serum TGF- $\beta$ levels, indicating susceptibility to the disease. The proinflammatory cytokines TNF- $\alpha$, IFN- $\gamma$, IL- $1 \beta$, IL-2, IL-5, and IL- 6 also showed high levels of expression during the infection period, suggesting that the balance between cytokines determines the course of infection. In DCs derived from BALB/c mice, we observed that no cytokine showed great peak expression; however, in C57BL/6, IL-10 and TNF- $\alpha$ were strongly stimulated by the Ninoa strain. The other cytokine levels did not change. Analyzing the serum of BALB/c animals infected with the Ninoa strain, the study conducted by Espinoza et al. demonstrated an increase in the levels of IL-10 in the acute phase, which decays and rises gradually during the course of infection. IL-12, which reached its peak in the acute phase and was maintained throughout the chronic phase, was also evaluated [17].

TLRs form a family of transmembrane proteins responsible for the recognition of molecular patterns essential for the triggering of immune responses [62-64]. Several surface molecules of T. cruzi are recognized by these receptors. Some molecules of trypomastigotes are recognized by TLR2 [65, $66]$, and other molecules present in epimastigotes are recognized by TLR4 [67]. The relationship between the recognition of T. cruzi by these receptors and the development of a susceptible or resistant immune response has been extensively investigated. TLR2, TLR4, TLR7, and TLR9 are impor- tant for the development mechanisms of T. cruzi infection [64]. The parasitic form tested in our study for all the strains used was the metacyclic, the same as that of the natural infections in vector transmission, and we observed a better expression of TLR2 in cells infected by the CL-Brener strain belonging to TcVI in relation to the INC5 strain belonging to TcI and a better expression of TLR4 in relation to the Ninoa strain in the TcI group. Similar studies using other strains showed strains belonging to the TcI group that were able to improve TLR2 expression, whereas the decrease in $\mathrm{TLR}^{+}$was evidenced in the TcII group [6]. The increase in TLR2 can be related to the ability to evade host defense by changing the cytokine profile produced, favoring the production of TLR2-dependent IL-10, as in infections by several other microorganisms $[6,51,59,67,68]$. Although this strain has not been highlighted by the production of IL-10 compared to others, the TLR2 pathway may be responsible for the observed production. TLR4 can trigger the production of cytokines such as IL-12, IFN- $\gamma$, TNF- $\alpha$, and nitric oxide (NO) in T. cruzi infections [67, 69], leading to better response. Our data show that the CL-Brener strain presents a greater number of TLR $4^{+}$DCs, although not showing a significant expression with respect to the cytokines of these profiles.

The ability of maturation and presentation of antigens by DCs is impaired by the presence of the parasite. This damage is due to the modulation of important molecules such as MHC, CD80, CD40, and CD86, whose levels are reduced in the presence of the parasite $[6,25,28,37,38]$. Only the INC5 strain induced high amounts of MHC-II. In general, this molecule is responsible for the success of antigen presentation and efficient assembly of specific immune response, including the production of antibodies [27, 30-32]. This is consistent with the data shown by Henrique et al., indicating that the most virulent strains Ninoa, INC5, and Colombian 
induced high production of antibodies. The costimulatory molecules CD80 and CD86 are also related to the maturation and presentation capacity of the antigen [27, 30-32, 52]. The strains studied here presented a negative variation for CD80 and CD86 in BMDCs derived from the two mouse lineages, suggesting a difficulty of maturation of these infected cells. A similar result was observed with splenic DCs infected with the Tehuantepec strain, where the decrease in CD86 expression prevented the migration of these cells to the antigenpresenting organs [47]. This represents a good escape strategy for the host defense parasite.

The metacyclic form is the infective form of transmission of T. cruzi, and the first cells found after infection are DCs. However, many studies on these cells were not performed using this infective form [6]. Thus, the evaluation of our dataset allows us to observe situations that can mimic what occurs during the interaction process with the host. By using strains of T. cruzi and different mice, we were able to expand the observations even further and conclude that the two Mexican strains studied here were capable of modulating the response of DCs, regardless of their origin, through different pathways. The results reinforce the escape strategy of the host immune response and demonstrate the importance of investigating the mechanisms involved in this process.

\section{Data Availability}

The datasets generated during and/or analyzed during the current study are available from the corresponding author on reasonable request.

\section{Disclosure}

The funders had no role in the study design, data collection and analysis, decision to publish, or preparation of the manuscript.

\section{Conflicts of Interest}

The authors declare that they have no conflicts of interest.

\section{Acknowledgments}

The research leading to these results has received support and funding from the Conselho Nacional de Desenvolvimento Científico e Tecnológico, Fundação de Amparo à Pesquisa do Estado de Minas Gerais (FAPEMIG), Coordenação de Aperfeiçoamento de Pessoal de Nível Superior (CAPES), and Network for Infectious Disease Research.

\section{References}

[1] C. Chagas, "Nova tripanozomiaze humana: estudos sobre a morfolojia e o ciclo evolutivo do Schizotrypanum cruzi $\mathrm{n}$. gen., n. sp., ajente etiolojico de nova entidade morbida do homem," Memórias do Instituto Oswaldo Cruz, vol. 1, no. 2, pp. 159-218, 1909.

[2] L. E. Echeverria and C. A. Morillo, "American trypanosomiasis (Chagas disease)," Infectious Disease Clinics of North America, vol. 33, no. 1, pp. 119-134, 2019.
[3] L. A. Messenger, J. D. Ramirez, M. S. Llewellyn, F. Guhl, and M. A. Miles, "Importation of hybrid human-associated Trypanosoma cruzi strains of southern South American origin, Colombia," Emerging Infectious Diseases, vol. 22, no. 8, pp. 1452-1455, 2016.

[4] B. Zingales, "Trypanosoma cruzi genetic diversity: something new for something known about Chagas disease manifestations, serodiagnosis and drug sensitivity," Acta Tropica, vol. 184, pp. 38-52, 2018.

[5] B. Zingales, S. Andrade, M. Briones et al., "A new consensus for Trypanosoma cruzi intraspecific nomenclature: second revision meeting recommends TcI to TcVI," Memórias do Instituto Oswaldo Cruz, vol. 104, no. 7, pp. 1051-1054, 2009.

[6] T. A. da Costa, M. V. Silva, M. T. Mendes et al., "Immunomodulation by Trypanosoma cruzi: toward understanding the association of dendritic cells with infecting TcI and TcII populations," Journal of Immunology Research, vol. 2014, Article ID 962047, 12 pages, 2014.

[7] N. Gil-Jaramillo, F. N. Motta, C. B. F. Favali, I. M. D. Bastos, and J. M. Santana, "Dendritic cells: a double-edged sword in immune responses during Chagas disease," Frontiers in Microbiology, vol. 7, article 1076, 2016.

[8] Z. Brener and E. Chiari, "Morphological variations observed in different strains of Trypanosoma cruzi," Revista do Instituto de Medicina Tropical de Sao Paulo, vol. 5, pp. 220-224, 1963.

[9] M. Cortez, M. R. Silva, I. Neira et al., "Trypanosoma cruzi surface molecule gp90 downregulates invasion of gastric mucosal epithelium in orally infected mice," Microbes and Infection, vol. 8, no. 1, pp. 36-44, 2006.

[10] I. Neira, F. A. Silva, M. Cortez, and N. Yoshida, "Involvement of Trypanosoma cruzi metacyclic trypomastigote surface molecule gp82 in adhesion to gastric mucin and invasion of epithelial cells," Infection and Immunity, vol. 71, no. 1, pp. 557-561, 2003.

[11] N. Yoshida, "Trypanosoma cruzi infection by oral route: how the interplay between parasite and host components modulates infectivity," Parasitology International, vol. 57, no. 2, pp. 105-109, 2008.

[12] "Recommendations from a satellite meeting," Memórias do Instituto Oswaldo Cruz, vol. 94, Supplement 1, pp. 429-432, 1999.

[13] C. I. Cura, A. M. Mejía-Jaramillo, T. Duffy et al., "Trypanosoma cruzi I genotypes in different geographical regions and transmission cycles based on a microsatellite motif of the intergenic spacer of spliced-leader genes," International Journal for Parasitology, vol. 40, no. 14, pp. 1599-1607, 2010.

[14] C. Herrera, M. D. Bargues, A. Fajardo et al., "Identifying four Trypanosoma cruzi I isolate haplotypes from different geographic regions in Colombia," Infection, Genetics and Evolution, vol. 7, no. 4, pp. 535-539, 2007.

[15] B. Zingales, M. A. Miles, D. A. Campbell et al., "The revised Trypanosoma cruzi subspecific nomenclature: rationale, epidemiological relevance and research applications," Infection, Genetics and Evolution, vol. 12, no. 2, pp. 240-253, 2012.

[16] C. G. Barbosa, C. Gómez-Hernández, K. Rezende-Oliveira et al., "Oral infection of mice and host cell invasion by Trypanosoma cruzi strains from Mexico," Parasitology Research, vol. 118, no. 5, pp. 1493-1500, 2019.

[17] B. Espinoza, T. Rico, S. Sosa et al., "Mexican Trypanosoma cruzi TCI Strains with Different Degrees of Virulence Induce Diverse Humoral and Cellular Immune Responses in a Murine 
Experimental Infection Model," Journal of Biomedicine and Biotechnology, vol. 2010, Article ID 890672, 10 pages, 2010.

[18] B. Espinoza, N. Solorzano-Domínguez, A. Vizcaino-Castillo, I. Martínez, A. L. Elias-López, and J. Rodríguez-Martínez, "Gastrointestinal infection with Mexican TcI Trypanosoma cruzi strains: different degrees of colonization and diverse immune responses," International Journal of Biological Sciences, vol. 7, no. 9, pp. 1357-1370, 2011.

[19] C. Gómez-Hernández, S. D. Pérez, K. Rezende-Oliveira et al., "Evaluation of the multispecies coalescent method to explore intra-Trypanosoma cruzi I relationships and genetic diversity," Parasitology, vol. 146, no. 8, pp. 1063-1074, 2019.

[20] C. Gómez-Hernández, K. Rezende-Oliveira, G. A. N. Nascentes et al., "Molecular characterization of Trypanosoma cruzi Mexican strains and their behavior in the mouse experimental model," Revista da Sociedade Brasileira de Medicina Tropical, vol. 44, no. 6, pp. 684-690, 2011.

[21] A. Vizcaíno-Castillo, A. Jimenez-Marin, and B. Espinoza, "Exacerbated skeletal muscle inflammation and calcification in the acute phase of infection by Mexican Trypanosoma cruzi DTUI strain," BioMed Research International, vol. 2014, Article ID 450389, 12 pages, 2014.

[22] J. R. R. Coura and A. C. Junqueira, "Surveillance, health promotion and control of Chagas disease in the Amazon Region - medical attention in the Brazilian Amazon Region: a proposal," Memórias do Instituto Oswaldo Cruz, vol. 110, no. 7, pp. 825-830, 2015.

[23] M. S. Llewellyn, M. D. Lewis, N. Acosta et al., “Trypanosoma cruzi IIc: phylogenetic and phylogeographic insights from sequence and microsatellite analysis and potential impact on emergent Chagas disease," PLoS Neglected Tropical Diseases, vol. 3, no. 9, 2009.

[24] B. A. de Noya, Z. Díaz-Bello, C. Colmenares et al., "Update on oral Chagas disease outbreaks in Venezuela: epidemiological, clinical and diagnostic approaches," Memórias do Instituto Oswaldo Cruz, vol. 110, no. 3, pp. 377-386, 2015.

[25] C. D. Alba Soto, M. E. Solana, C. V. Poncini, A. M. Pino-Martinez, V. Tekiel, and S. M. González-Cappa, "Dendritic cells devoid of IL-10 induce protective immunity against the protozoan parasite Trypanosoma cruzi," Vaccine, vol. 28, no. 46, pp. 7407-7413, 2010.

[26] E. J. Pearce and B. Everts, "Dendritic cell metabolism," Nature Reviews Immunology, vol. 15, no. 1, pp. 18-29, 2014.

[27] S. Hugues, "Dynamics of dendritic cell-T cell interactions: a role in T cell outcome," Seminars in Immunopathology, vol. 32, no. 3, pp. 227-238, 2010.

[28] S. P. Manickasingham, A. . D. Edwards, O. Schulz, and C. Reis e Sousa, "The ability of murine dendritic cell subsets to direct T helper cell differentiation is dependent on microbial signals," European Journal of Immunology, vol. 33, no. 1, pp. 101-107, 2003.

[29] O. Takeuchi and S. Akira, "Pattern recognition receptors and inflammation," Cell, vol. 140, no. 6, pp. 805-820, 2010.

[30] J. S. Blum, P. A. Wearsch, and P. Cresswell, "Pathways of antigen processing," Annual Review of Immunology, vol. 31, no. 1, pp. 443-473, 2013.

[31] M. F. Lipscomb and B. J. Masten, "Dendritic cells: immune regulators in health and disease," Physiological Reviews, vol. 82, no. 1, pp. 97-130, 2002.

[32] B. M. O'Neill, D. Hanway, E. A. Winzeler, and F. E. Romesberg, "Coordinated functions of WSS1, PSY2 and
TOF1 in the DNA damage response," Nucleic Acids Research, vol. 32, no. 22, pp. 6519-6530, 2004.

[33] R. Förster, A. Schubel, D. Breitfeld et al., "CCR7 coordinates the primary immune response by establishing functional microenvironments in secondary lymphoid organs," Cell, vol. 99, no. 1, pp. 23-33, 1999.

[34] S. R. Holdsworth and P.-Y. Y. Gan, "Cytokines: names and numbers you should care about," Clinical journal of the American Society of Nephrology, vol. 10, no. 12, pp. 2243-2254, 2015.

[35] V. Verhasselt, C. Buelens, F. Willems, D. De Groote, N. Haeffner-Cavaillon, and M. Goldman, "Bacterial lipopolysaccharide stimulates the production of cytokines and the expression of costimulatory molecules by human peripheral blood dendritic cells: evidence for a soluble CD14-dependent pathway," The Journal of Immunology, vol. 158, no. 6, pp. 2919-2925, 1997.

[36] F. R. S. Gutierrez, F. S. Mariano, C. J. F. Oliveira et al., "Regulation of Trypanosoma cruzi-induced myocarditis by programmed death cell receptor 1," Infection and Immunity, vol. 79, no. 5, pp. 1873-1881, 2011.

[37] C. V. Poncini, C. D. Alba Soto, E. Batalla, M. E. Solana, and S. M. González Cappa, "Trypanosoma cruzi induces regulatory dendritic cells in vitro," Infection and Immunity, vol. 76, no. 6, pp. 2633-2641, 2008.

[38] C. V. Poncini, J. M. Ilarregui, E. I. Batalla et al., “Trypanosoma cruzi Infection Imparts a Regulatory Program in Dendritic Cells and T Cells via Galectin-1-Dependent Mechanisms," The Journal of Immunology, vol. 195, no. 7, pp. 3311-3324, 2015.

[39] C. D. Alba Soto, G. A. Mirkin, M. E. Solana, and S. M. González Cappa, "Trypanosoma cruzi infection modulates in vivo expression of major histocompatibility complex class II molecules on antigen-presenting cells and T-cell stimulatory activity of dendritic cells in a strain-dependent manner," Infection and Immunity, vol. 71, no. 3, pp. 11941199, 2003.

[40] W. O. Dutra, C. A. S. Menezes, L. M. D. Magalhães, and K. J. Gollob, "Immunoregulatory networks in human Chagas disease," Parasite Immunology, vol. 36, no. 8, pp. 377-387, 2014.

[41] P. M. Nogueira, K. Ribeiro, A. C. O. Silveira et al., "Vesicles from different Trypanosoma cruzi strains trigger differential innate and chronic immune responses," Journal of Extracellular Vesicles, vol. 4, no. 1, article 28734, 2015.

[42] L. Planelles, M. C. Thomas, C. Marañón, M. Morell, and M. C. López, "Differential CD86 and CD40 co-stimulatory molecules and cytokine expression pattern induced by Trypanosoma cruzi in APCs from resistant or susceptible mice," Clinical and Experimental Immunology, vol. 131, no. 1, pp. 41-47, 2003.

[43] C. V. Poncini and S. M. González-Cappa, "Dual role of monocyte-derived dendritic cells in Trypanosoma cruzi infection," European Journal of Immunology, vol. 47, no. 11, pp. 1936-1948, 2017.

[44] V. M. Monteon, J. Furuzawa-Carballeda, R. Alejandre-Aguilar, A. Aranda-Fraustro, J. L. Rosales-Encina, and P. A. Reyes, "American trypanosomosis: in situ and generalized features of parasitism and inflammation kinetics in a murine model," Experimental Parasitology, vol. 83, no. 3, pp. 267-274, 1996.

[45] R. Ruíz-Sánchez, M. P. León, V. Matta et al., "Trypanosoma cruzi isolates from Mexican and Guatemalan acute and chronic chagasic cardiopathy patients belong to Trypanosoma 
cruzi I," Memórias do Instituto Oswaldo Cruz, vol. 100, no. 3, pp. 281-283, 2005.

[46] M. M. Teixeira and N. Yoshida, "Stage-specific surface antigens of metacyclic trypomastigotes of Trypanosoma cruzi identified by monoclonal antibodies," Molecular and Biochemical Parasitology, vol. 18, no. 3, pp. 271-282, 1986.

[47] D. Chaussabel, B. Pajak, V. Vercruysse et al., "Alteration of migration and maturation of dendritic cells and T-cell depletion in the course of experimental Trypanosoma cruzi infection," Laboratory Investigation, vol. 83, no. 9, pp. 1373-1382, 2003.

[48] S. Malaga and N. Yoshida, "Targeted reduction in expression of Trypanosoma cruzi surface glycoprotein gp90 increases parasite infectivity," Infection and Immunity, vol. 69, no. 1, pp. 353-359, 2001.

[49] N. Yoshida, "Molecular basis of mammalian cell invasion by Trypanosoma cruzi," Anais da Academia Brasileira de Ciencias, vol. 78, no. 1, pp. 87-111, 2006.

[50] J. de Meis, J. Barreto de Albuquerque, D. Silva dos Santos et al., "Trypanosoma cruzi entrance through systemic or mucosal infection sites differentially modulates regional immune response following acute infection in mice," Frontiers in Immunology, vol. 4, p. 216, 2013.

[51] B. L. Ferreira, É. R. Ferreira, M. V. de Brito et al., "BALB/c and C57BL/6 mice cytokine responses to Trypanosoma cruzi infection are independent of parasite strain infectivity," Frontiers in Microbiology, vol. 9, p. 553, 2018.

[52] P. M. Henrique, T. Marques, M. V. da Silva et al., "Correlation between the virulence of T. cruzi strains, complement regulatory protein expression levels, and the ability to elicit lytic antibody production," Experimental Parasitology, vol. 170, pp. 6672,2016

[53] B. A. Burleigh and N. W. Andrews, "Signaling and host cell invasion by Trypanosoma cruzi," Current Opinion in Microbiology, vol. 1, no. 4, pp. 461-465, 1998.

[54] R. A. Mortara, "Trypanosoma cruzi: amastigotes and trypomastigotes interact with different structures on the surface of HeLa cells," Experimental Parasitology, vol. 73, no. 1, pp. 1-14, 1991.

[55] F. Y. Maeda, T. M. Clemente, S. Macedo, C. Cortez, and N. Yoshida, "Host cell invasion and oral infection by Trypanosoma cruzi strains of genetic groups TcI and TcIV from chagasic patients," Parasites \& Vectors, vol. 9, no. 1, p. 189, 2016.

[56] R. C. Ruiz, S. Favoreto, L. M. Dorta et al., "Infectivity of Trypanosoma cruzi strains is associated with differential expression of surface glycoproteins with differential Ca2+ signalling activity," Biochemical Journal, vol. 330, no. 1, pp. 505-511, 1998.

[57] S. C. Goncalves da Costa, K. S. Calabrese, T. Zaverucha do Valle, and P. H. Lagrange, "Trypanosoma cruzi: infection patterns in intact and athymic mice of susceptible and resistant genotypes," Histology and Histopathology, vol. 17, no. 3, pp. 837-844, 2002.

[58] E. Roggero, A. Perez, M. Tamae-Kakazu et al., "Differential susceptibility to acute Trypanosoma cruzi infection in BALB/c and $\mathrm{C} 57 \mathrm{BL} / 6$ mice is not associated with a distinct parasite load but cytokine abnormalities," Clinical \& Experimental Immunology, vol. 128, no. 3, pp. 421-428, 2002.

[59] P. S. Doyle, Y. M. Zhou, I. Hsieh, D. C. Greenbaum, J. H. McKerrow, and J. C. Engel, "The Trypanosoma cruzi protease cruzain mediates immune evasion," PLoS Pathogens, vol. 7, no. $9,2011$.
[60] C. A. Hunter, L. A. Ellis-Neyes, T. Slifer et al., "IL-10 is required to prevent immune hyperactivity during infection with Trypanosoma cruzi," The Journal of Immunology, vol. 158, no. 7, pp. 3311-3316, 1997.

[61] J. S. Silva, D. R. Twardzik, and S. G. Reed, "Regulation of Trypanosoma cruzi infections in vitro and in vivo by transforming growth factor beta (TGF-beta)," The Journal of Experimental Medicine, vol. 174, no. 3, pp. 539-545, 1991.

[62] T. Kawai and S. Akira, "The role of pattern-recognition receptors in innate immunity: update on Toll-like receptors," Nature Immunology, vol. 11, no. 5, pp. 373-384, 2010.

[63] E. B. Kopp and R. Medzhitov, "The Toll-receptor family and control of innate immunity," Current opinion in immunology, vol. 11, no. 1, pp. 13-18, 1999.

[64] M. M. Rodrigues, A. C. Oliveira, and M. Bellio, "The immune response to Trypanosoma cruzi: role of toll-like receptors and perspectives for vaccine development," Journal of Parasitology Research, vol. 2012, Article ID 507874, 12 pages, 2012.

[65] I. C. Almeida and R. T. Gazzinelli, "Proinflammatory activity of glycosylphosphatidylinositol anchors derived from Trypanosoma cruzi: structural and functional analyses," Journal of Leukocyte Biology, vol. 70, no. 4, pp. 467-477, 2001.

[66] M. A. S. Campos, I. C. Almeida, O. Takeuchi et al., "Activation of Toll-like receptor-2 by glycosylphosphatidylinositol anchors from a protozoan parasite," The Journal of Immunology, vol. 167, no. 1, pp. 416-423, 2001.

[67] A. C. Oliveira, J. R. Peixoto, L. B. de Arruda et al., "Expression of functional TLR4 confers proinflammatory responsiveness to Trypanosoma cruzi glycoinositolphospholipids and higher resistance to infection with T. cruzi," The Journal of Immunology, vol. 173, no. 9, pp. 5688-5696, 2004.

[68] D. E. Gaddis, C. L. Maynard, C. T. Weaver, S. M. Michalek, and J. Katz, "Role of TLR2-dependent IL-10 production in the inhibition of the initial IFN- $\gamma$ T cell response to Porphyromonas gingivalis," Journal of Leukocyte Biology, vol. 93, no. 1, pp. 21-31, 2013.

[69] C. H. Kim, L. S. Rott, I. Clark-Lewis, D. J. Campbell, L. Wu, and E. C. Butcher, "Subspecialization of Cxcr5 ${ }^{+}$T cells," Journal of Experimental Medicine, vol. 193, no. 12, pp. 1373-1382, 2001. 


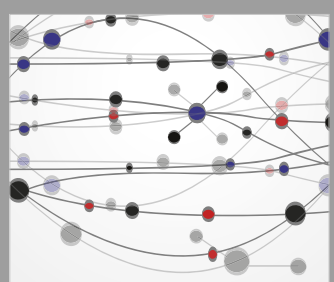

The Scientific World Journal
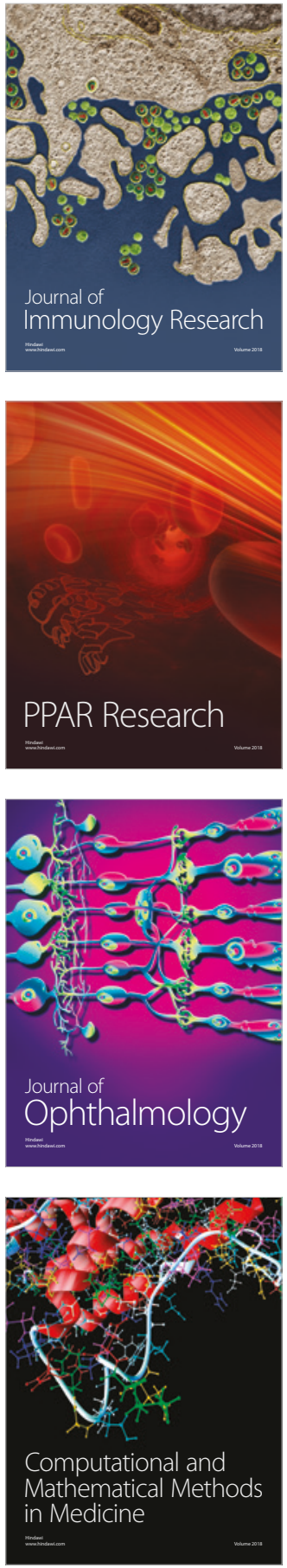

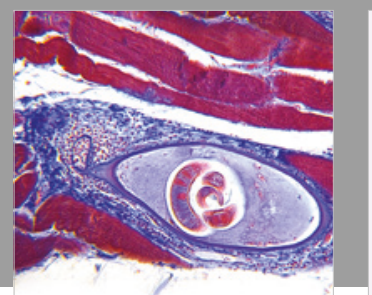

Gastroenterology Research and Practice

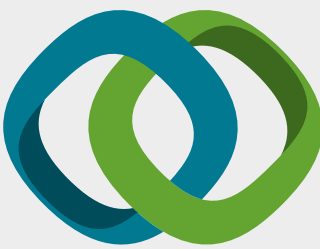

\section{Hindawi}

Submit your manuscripts at

www.hindawi.com
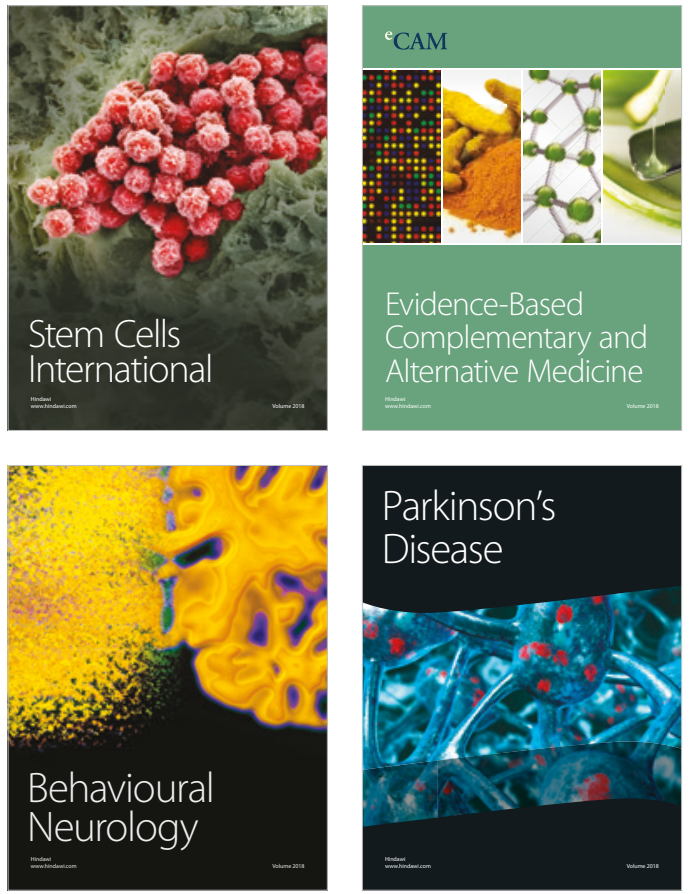

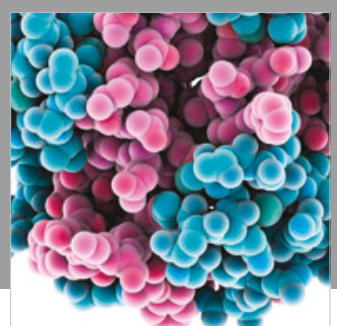

ournal of

Diabetes Research

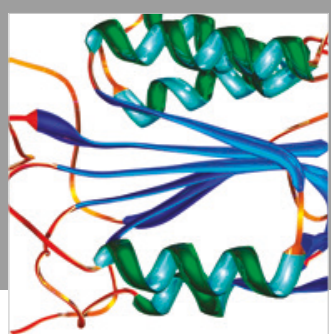

Disease Markers
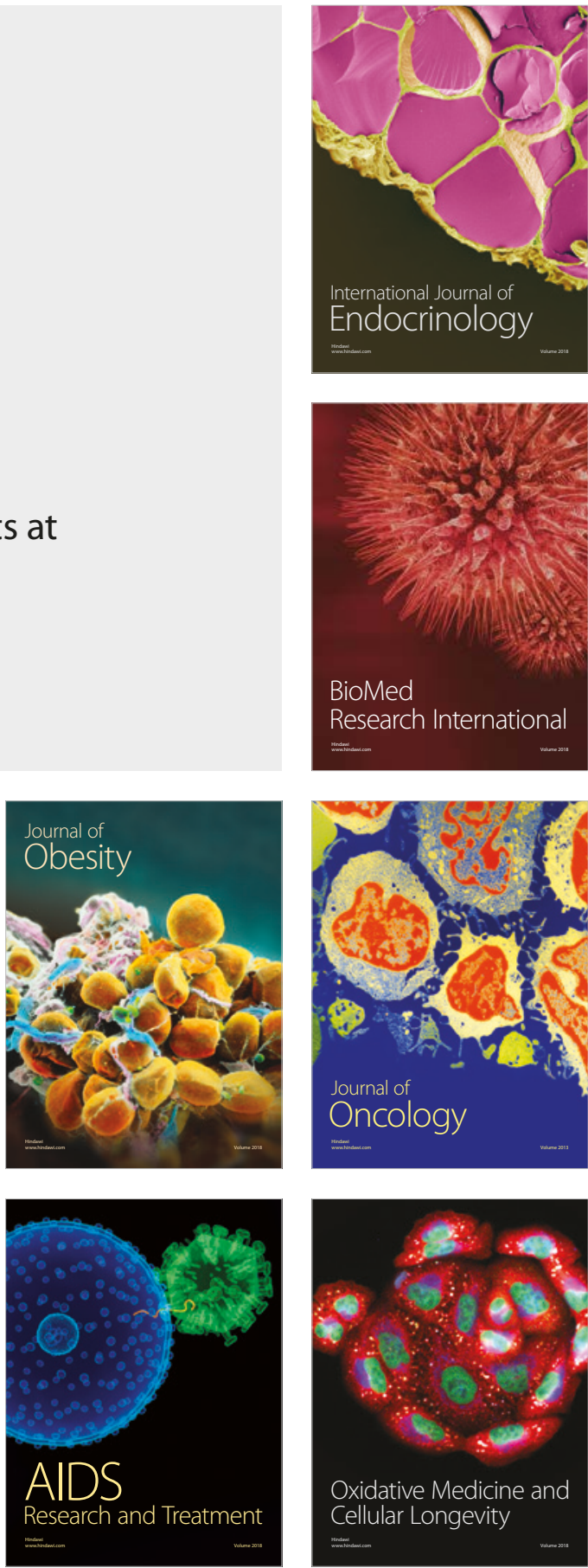